\title{
Sudden Unexpected Death in Epilepsy: a Case Scenario to elaborate the need of awareness and a protocol for local setup
}

\author{
Ariyarathna HTDW, Hulathduwa SR* \\ Department of Forensic Medicine, Faculty of Medical Sciences, University of Sri Jayewardenepura, Nugegoda, \\ Sri Lanka.
}

\begin{abstract}
Sudden unexpected death in epilepsy (SUDEP) is the commonest cause of death related to epilepsy. There is no settled proposition among neurologists, neuropathologists and forensic pathologists as to the exact pathophysiology of SUDEP. Though there is renowned interest about this entity in the developed world, there is little awareness and interest about SUDEP in the developing world. In the developed countries there are established SUDEP awareness programmes run by the state and other scientific organizations. They help in increasing the awareness of this entity among the general public, ensuring safety and well-being of the diagnosed epileptic patients and updating the knowledge among the clinicians and other academics. It is the authors' view that the judicial medical officers in Sri Lanka too owe a similar responsibility and obligation towards the clinicians and the general public regarding raising the awareness of SUDEP and establishing a clinico-pathologic correlation. Diagnosis of SUDEP is a challenge in forensic pathology. It needs careful exclusion of suicidal, homicidal and accidental circumstances as well as other possible natural causes. This warrants careful study of the scene, history from several sources, meticulous autopsy examination followed by routine and ancillary investigations. We present a case report of SUDEP highlighting some of the deficiencies in Sri Lanka including the absence of a protocol to ensure quality-assured minimal standards in the investigation of suspected SUDEP cases.
\end{abstract}

Keywords: Anti-epileptic drugs, negative autopsy, sudden deaths, SUDEP awareness, SUDEP protocol

Received: 20 June 2020, Revised version accepted: 29 June 2020, Published: 30 June 2020. *Corresponding author: Hulathduwa SR, $\bigotimes$ Email: ariyaratna@sjp.ac.lk (D) https://orcid.org/0000-0002-4266-5508

Cite this article as: Ariyarathna HTDW, Hulathduwa SR. Sudden Unexpected Death in Epilepsy: a Case Scenario to elaborate the need of awareness and a protocol for local setup. Medico-Legal Journal of Sri Lanka, 2020; 8(1): 33-37. DOI: http://doi.org/10.4038/mljsl.v8i1.7408

Copyright: @ 2019 with the Medico-legal Journal of Sri Lanka.

This is an open-access article distributed under the terms of the Creative Commons Attribution 4.0 International License, which permits unrestricted use, distribution and reproduction in any medium provided the original author and source are credited.

\section{Introduction}

Sudden and unexpected death of an epileptic patient which is not attributable to secondary causes such as drowning, burns, different forms of accidents etc. or to other forms of natural illnesses such as ischaemic heart disease or pneumonia, but solely due to epilepsy is designated as SUDEP.[1] The percentage of such deaths is around $2-17 \%$ among the epileptics. ${ }^{[1]}$ This roughly amounts to $1-2$ deaths per 1000 epileptic patients per year globally. ${ }^{[2]}$ SUDEP remained a diagnostic and therapeutic challenge over several decades. Disciplines of neurology, neuropathology and forensic pathology should work collaboratively in order to investigate suspected SUDEPs. ${ }^{[1]}$ Until early 1990s the public awareness of this condition was very poor and the medical literature too was scanty. During that time it was not recognized as a distinct entity which can be denoted as an acceptable cause of death. ${ }^{[3]}$ Children and the young adults have been identified as the most vulnerable. It is the authors' personal view and understanding that the awareness of SUDEP is extremely poor even among the immediate family members of epileptic patients in Sri Lanka. A study of Massey et. al. revealed that the awareness and the interest of this condition among the health care providers including clinicians are not adequate even in the developed world. ${ }^{[4]}$ Compared to the normal population, an increased mortality rate had been identified among epileptics even without established evidence of an epileptic attack prior to death. ${ }^{[5]}$ Because of this sudden and unexpected nature of the death, a formal inquiry prior to the disposal of the body should be carried out according to Sri Lankan jurisdiction. It is most advisable that the inquirer into sudden deaths directs such deaths to a forensic pathologist who will perform the final multispectral scientific investigation with an attempt to solve at least the possible medico-legal issues pertaining to such deaths. This approach is beneficial to multiple 
parties including the deceased, his relatives, the clinicians and the pathologist as well as the general public as it helps to sort out their problems and issues while building up a clinico-pathologic correlation. It also rules out possible fowl-play providing the deceased with justice which is his due.

\section{Case report}

The deceased was a 32-year-old known epileptic female. She was unmarried as she had been mentally subnormal and epileptic since early childhood. She had been fully investigated by several consultant paediatricians, neurologists and psychiatrists during early childhood where no demonstrable cause was found either for her epilepsy or mental retardation. Furthermore a link between mental unsoundness and epilepsy had also not been established. She had been on regular treatment and follow-up for Grand Mal epilepsy alone, the mental unsoundness been left alone as it was not progressive or disturbing to her life as well as the lives of her associates. Her drug compliance was satisfactory. Proceeding an apparently uneventful period, one morning she was found dead in prone position on her bed. The scene was undisturbed. The mother of the deceased was also sleeping in the same room but on a separate bed. She had noticed that the deceased had passed urine during sleep, which she does only during an epileptic attack. The scene visit was unremarkable.

The post-mortem examination with special neck, musculoskeletal and neuropathological dissections revealed no external or internal injuries contributory to or causative of the death except for the fresh tongue bites highly suggestive of an epileptic attack prior to death. The clothes were soaked with urine. All internal organs including the brain appeared normal. No cerebral oedema was noted. Further, there were no space occupying lesions, vascular malformations or previous infarctions. The heart was unremarkable. There were no areas of myocardial fibrosis or any other anatomical or pathological abnormality. The lungs showed mild pulmonary oedema. Samples were collected for histoplathological examination. These included multiple samples from different sites of the brain (hippocampus, cerebral cortex, basal ganglia, cerebellum etc.), all four chambers of the heart, lungs, kidneys and all the other important organs. Hypocampal sclerosis or eosinophilic neurons were not noted. The toxicology report was unremarkable. Blood was negative for anti-epileptic drugs. After careful consideration of the overall history and the autopsy findings, the cause of death was given as SUDEP. The relatives were unaware of a condition termed SUDEP and its higher tendency for sudden unexpected natural deaths. Advice on close observation, use of alarms, 'anti-suffocation' pillows etc. had not been received by the relatives or the care-givers.

\section{Discussion}

The recognition of SUDEP demands a higher level of suspicion and vigilance among death investigators. This is equally true for Sri Lanka. The definition of SUDEP lacks consensus among different authorities. One widely accepted definition (first proposed by Nashef et. al ) is as follows: "Sudden, unexpected, witnessed or unwitnessed, non-traumatic and non-drowning death in patients with epilepsy, with or without evidence of a seizure and excluding documented status epilepticus, in which post-mortem examination does not reveal a toxicological or anatomic cause for death." [6] This definition is almost twenty two years old though it is still widely accepted.

The following criteria should be fulfilled for a death to be labeled as SUDEP. (1) Previously diagnosed epileptic patient, probably with recurrent, unprovoked seizures, (2) Apparently healthy person dies unexpectedly with no other cause, (3) Should be a sudden death and (4) There may or may not be evidence of seizure, but status epilepticus should be excluded. ${ }^{[7]}$ According to above guidelines it is evident that this case under discussion undoubtedly falls in to SUDEP. Some authorities have divided SUDEP further into three sub-categories. The basis of this classification is the degree of certainty of the diagnosis. ${ }^{[8]}$ Definitive SUDEP is a death of an epileptic with a compatible clinical picture where no alternative cause of death had been revealed at the autopsy. A probable SUDEP is a death for which no autopsy had been performed but all the clinical criteria had been met with while a possible SUDEP is where the circumstances leading to death are inconclusive to ascertain the death as SUDEP while no postmortem had been performed.

In clinical set up, if a patient with a known history of epilepsy was successfully resuscitated while having a cardio-pulmonary arrest in consequence of an epileptic attack; such an event is termed nearSUDEP. ${ }^{[9]}$

Many risk factors have been identified. Some are modifiable though the rest is non-modifiable. The most important clinical risk factor is the frequency of tonic clonic seizures. ${ }^{[10]}$ Death during sleep and nocturnal seizures prior to death had been shown to be present in $70 \%$ of cases studied in certain largescale studies. ${ }^{[11]}$ Age being less than 40 years and sleeping in prone (face down) position are also identified as risk factors. ${ }^{[12]}$ Having nocturnal seizures, longer seizure duration, younger onset of the first episode of seizure, multiple anti-epileptic drug therapy, poor seizure control despite optimal drug therapy and periods of lack of proper monitoring too have been identified as additional 
risk factors. All above except the male gender were present in the case under discussion. Additionally it had been identified that being mentally subnormal is also one of the significant risk factors which too was present in the deceased. ${ }^{[13]}$

The benefit of identifying risk factors in SUDEP is multiple. It gives the clinicians, the patient, his care givers and family members an insight as to the level of risk the patient is carrying. This enables the patient and the care givers to optimize the behavioral patterns and clinicians to advice the patient on changing modifiable risk factors. Recently, certain authors have attempted to stratify the risk factors in order to place each patient on a 'risk score'. This score or the index will provide a clue to the patient's proneness to sudden and unexpected death attributable to epilepsy. ${ }^{[14]}$ The awareness made among the patients and their care-givers would enable to minimize preventable and premature deaths from being taken place. Controversy exists in some counties as whether to reveal or not about this outcome to the caregivers. ${ }^{[15]}$ In Sri Lankan context, the authors believe that the best approach is the public awareness programmes launched in a wide scale in order to modify certain risk factors.

The 23rd of October had been announced globally as the "SUDEP awareness day." In developed countries there are established government and nongovernmental professional organizations working together and individually towards promoting public awareness of SUDEP and aiding professionals on further research on this topic. Epilepsy Foundation, SUDEP Action, Epilepsy Society and Epilepsy Ontario are few among many such to mention. It is the authors' perception that Sri Lanka is lagging behind in this context, the awareness of SUDEP among the clinicians, the epileptic patients, the general public and the death investigators including the judicial medical officers being not satisfactory. The authors have come to this conclusion after indepth qualitative analysis of the few SUDEP cases they have performed during the past seven years including the case under discussion which is the most recent. This had been done as a part of internal quality assurance process. SUDEP is still a concept not deeply ingrained in the minds of the death investigators in Sri Lanka. Thus, there is a possibility that true SUDEP cases be over-looked making the entity being under-reported. Clinicians treating epileptic patients should take effort to identify high-risk clients for SUDEP and act accordingly. Once an epileptic death is reported in hospital setup, measures should be taken for thorough investigation. In the recent past, the Director General of Health Services has passed circulars mandating all Dengue and Maternal Deaths to be subjected to inquest. The authors suggest that it will be a step forward in preventing SUDEP if all epileptic deaths be subject to inquest and thereafter the more ambiguous cases be directed for a judicial autopsy. As there is no such provision at present mandating an inquest on all epileptic deaths, clinicians should take the initiative to direct such ward-deaths for inquest or if the cause of death and other circumstances are too obvious for the death to be directed for inquest, they could request from the legal guardian of the body the permission for a pathological autopsy in which a thorough neuropathological dissection and sampling could be achieved. As per the judicial autopsies, medical officers of all calibers performing judicial autopsies should possess adequate awareness, knowledge, skills as well as the positive attitude of performing an extensive and complete autopsy on putative/prospective SUDEP cases. In the absence of adequate facilities to rule out other causes, such cases are best directed to centres with better facilities and a specialist in Forensic Medicine.

In the context of prevention of SUDEP, as well as in delineating the causes of death at a suspected SUDEP autopsy, a basic understanding of the wide spectrum of mechanisms postulated for the sudden and unexplained deaths among epileptics is vital. A cascade of events had been speculated over the years in autonomic nervous, respiratory and cardiac systems in genetically susceptible individuals with unique genomic complexities or mutations. Yet, the exact cause/causes/mechanism playing the major role in such deaths is still obscure. Establishment of cardio-autonomic and respiratory dysfunctions has been currently identified as the principal mechanisms causing SUDEP. ${ }^{[16]}$ Recently, attention had been focused on molecular profiling and genetic studies including novo mutations leading to cardiac ion channel dysfunctions affecting the neurocardiac, neuroautonomic and neurorespiratory pathways. Debates are still on among neuropathologists, neurologists and researchers as to what the most common primary/initiative/triggering event is. ${ }^{[17]} \mathrm{It}$ could well be cardiac, respiratory or both. Changes in blood pressure with asystole led to the assumption that SUDEP is due to cardiac malfunctioning mechanisms. Similarly, respiratory depression and apnoea occurring during and after a seizure episode causing oxygen desaturation are two other principle mechanisms postulated by some authorities. Compromised normal arousal mechanisms had been identified in these patients as another mechanism possible to cause death. Another significant mechanism with renowned interest since recent past is obstructive laryngeal spasms with subsequent respiratory arrest. Irritation of the larynx with acid regurgitation from the stomach during an episode of seizure is currently thought as the reason for laryngeal spasm though inherent (spontaneous) pathology of the recurrent laryngeal nerve was the former (older) postulation for the triggering 
mechanism. ${ }^{[18]}$ There are numerous other intricate speculations for the mechanisms and pathophysiology of SUDEP. The field is ever growing and more and more research is being done in the developed world so as to achieve a better insight to the triggering mechanisms of SUDEP.

Sudden and unexpected loss of a family member is a serious and perhaps rather catastrophic event in any socio-cultural setting. Yet, there is much to do both in the community set up as well as in the hospital set up (clinics and wards) to educate the patients and their immediate family members regarding the increased risk of accidents, injuries and death this group of patients are carrying with them and the practical measures to prevent and minimize the outcome of the same. Divulging the fact of increased proneness to sudden deaths may initially be depressive to the recipient though the authors wish that benefits of telling so will far outweigh its negative effects in the long run. Further, every patient has his right to know the risks and outcome of his disease condition as well as the measures to be taken to prevent them. Many researchers have highlighted that lack of night time supervision is one of the easily modifiable risk factors. ${ }^{[19]}$

Inquiring in to this fact during the pre-autopsy history taking in the present case as well as in all the other cases the two authors have performed in Sri Lanka for the past seven years revealed that the principle care-giver (the mother of the deceased in the present case) was unaware and had never been informed of this simple measure. The same was asked from the family members of two different SUDEP subjects whose autopsies had been done at the Victorian Institute of Forensic Medicine (VIFM) Australia, by one of the authors during the postautopsy discussions with family members. They were well informed by the attending neurologist as well as their general practitioner of the necessary facts and figures as well as the myths revolving around SUDEP. They also have participated in several SUDEP awareness programmes organized by the local government.

There are basically two levels of medical officers who have to formulate the cause of death (COD) according to the WHO format, namely the forensic pathologists and the ward doctors. Forensic pathologists should pay extra attention in formulating the COD as it will be contributed to national statistics and further implementation of national health and social policy planning. Though SUDEP accounts for up to $17 \%$ of all epilepsyrelated deaths and up to $50 \%$ of all deaths in refractory epilepsy, amounting to deaths of 1 in 1000 adults and 1 in 4500 children with epilepsy annually, some authors believe that it is too "non-specific" a terminology for a cause of death. ${ }^{[20]}$ This view had been expressed by the American Epilepsy Society in 2014. [21] 2019 ICD-10-CM Diagnosis Code G 40.909 is given to designate "Epilepsy-unspecified" without status epilepticus. This should denote the entity of SUDEP. ${ }^{[22]}$ The authors too share the same stance that SUDEP is not a very informative cause of death. Yet, it collectively designates the nonspecific group of natural deaths in epileptics which are not attributable to any other cause including status epilepticus but occurring by virtue of the deceased being an epileptic. The intricate neuropathophysiology behind the various mechanisms postulated by different authorities is mostly of high academic value.

If an epileptic dies of an unnatural cause such as drowning, fall off a height, road trauma or burns, the underlying cause should be mentioned as "epilepsy". In the same way, in all SUDEP deaths, the underlying cause should also be mentioned as "epilepsy". Misdiagnosis, under-diagnosis and overdiagnosis are all possible in SUDEPS. Furthermore, there is no consensus among the Sri Lankan forensic fraternity as to what the basic minimal standards to be adhered to are when conducting an autopsy on SUDEP in the context of history taking, scene visit, examination, dissection and investigations etc. As such, the authors wish to highlight the timely need for a protocol for SUDEP autopsies, practically feasible to be adhered to by all doctors engaged in medico-legal practice island-wide. This will enable to minimize diagnostic errors and pave path for quality-assured forensic audit in SUDEP.

It was able to reduce the mortality of Sudden Infant Deaths Syndrome (SIDS) by safe sleeping habits and other life-style modifications aided by the widespread awareness programmes targeting the general public and specially the mothers of the newborn. Scientists and researchers have opined that SIDS and SUDEP share many similarities as a "sudden death syndrome" though their physiological causes are poorly understood and possibly diverse. ${ }^{[23]}$ Therefore the possibility of reduction of SUDEP through mass scale public awareness programmes and behaviour modifications is a positive approach which had been tried in the developed world with promising results. Interested parties should pay attention to implement this in Sri Lanka as well.

\section{Conclusions}

Epileptics show an increased mortality compared to general population due to varied causes. SUDEP is one such significant cause. SUDEP confronts the clinician and the pathologist with many issues. Misdiagnosis, under-diagnosis and over-diagnosis are all possible. If the pathologist adheres to a 
national-level protocol in conducting the autopsy so as to ensure that the minimal standards are meted with, that will enable to collect accurate national statistics on SUDEP which intern will help to recognize the patterns and implement preventive measures. It also will ensure a clinico-pathologic correlation.

\section{Disclosure statement}

Conflicts of interests: The authors declare that they have no conflicts of interests.

\section{Funding: None}

\section{References}

1. Esen Mlez I, Arslan M, , Melez D, Sanli A, Koc S. Sudden Unexpected Death in Epilepsy: A Retrospective Autopsy Study of 112 Epileptic Patients. Noro Psikiyatri Arsivi. 2017;54(3): 225-33.

2. Thurman DJ, Hesdorffer DC, French JA. Sudden unexpected death in epilepsy: Assessing the public health burden. Epilepsia. 2014;55(10):1479-85.

3. Jones L, Thomas R. Sudden death in epilepsy: Insights from the last 25 years. Seizure. 2017; 44(2) : 232-6.

4. Massey C, Sowers L, Dlouhy B, Richerson G. Mechanisms of sudden unexpected death in epilepsy: the pathway to prevention. Nature Reviews Neurology. 2014;10(5): 271-82.

5. Fisher RS, Boas WvE, Blume W, Elger C, Genton P, Lee P, Engel J. Epileptic seizures and epilepsy: definitions proposed by the International League Against Epilepsy (ILAE) and the International Bureau for Epilepsy (IBE)

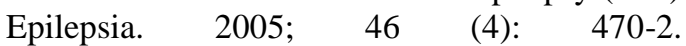
https://doi.org/10.1111/j.00139580.2005.66104.x.

6. Nashef L. Sudden unexpected death in epilepsy: terminology and definitions. Epilepsia 1997; 38(Suppl. 11):S6-8.

7. Sperling M. Sudden Unexplained Death in Epilepsy. Epilepsy Currents. 2001; 1(1): 21-3.

8. Annegers JF, Coan SP. SUDEP: overview of definitions and review of incidence data. Seizure 1999;8(6):347-52.

9. Nashef L, So EL, Ryvlin P, Tomson T. Unifying the definitions of sudden unexpected death in epilepsy. Epilepsia. 2012; 53 (4): 227-33.

10. Tomson T, Surges R, Delamont R, Haywood S, Hesdorffer D. Who to target in sudden unexpected death in epilepsy prevention and how? Risk factors, biomarkers, and intervention study designs. Epilepsia. 2016; 57(4) :4-16.

11. Ali, A., Wu, S., Issa, N.P. et al. Association of sleep with sudden unexpected death in epilepsy. Epilepsy Behav. 2017; 76 (4) : 1-6.
12. Manolis T, Manolis A, Melita H, Manolis A. Sudden unexpected death in epilepsy: The neurocardio-respiratory connection. Seizure. 2019;64(3):65-73.

13. Rowan, A.J. Seizure Prognosis in Long-Stay Mentally Subnormal Epileptic Patients: Interrater EEG and Clinical Studies. [Online]. Available from: https://pubmed.ncbi.nlm.nih.gov/7379766 [Accessed on 25/05/2020].

14. Shankar R, Newman C, McLean B. Has the Time Come to Stratify and Score SUDEP Risk to Inform People With Epilepsy of Their Changes in Safety? Frontiers in Neurology. 2018; 9.

15. Friedman D, Donner E, Stephens D, Wright C, Devinsky O. Sudden unexpected death in epilepsy: Knowledge and experience among U.S. and Canadian neurologists. Epilepsy \& Behavior. 2014;35 (3):13-8.

16. Goldman A. Mechanisms of sudden unexplained death in epilepsy. Current Opinion in Neurology. 2015; 28(2): 166-74.

17. Coll M, Oliva A, Grassi S, Brugada R, Campuzano O. Update on the Genetic Basis of Sudden Unexpected Death in Epilepsy. International Journal of Molecular Sciences. 2019;20(8):197-9.

18. Budde R, Arafat M, Pederson D, Lovick T, Jefferys J, Irazoqui P. Acid reflux induced laryngospasm as a potential mechanism of sudden death in epilepsy. Epilepsy Research 2018: 148(4):23-31.

19. Patients Who Die of SUDEP Largely Live Alone and Die Unwitnessed at Home Mdedge.com. Neurology Reviews. 2018 June;26(6):40

20. Shorvon S, Trinka E. Status epilepticus-Making progress. Epilepsia; 2011. 55(10):1235-8.

21. Aesnet.org. 2019 https://www.aesnet.org/sites/default/files/file_at tach/Meeting/Press/Changing\%20Communicati on\%20Around\%20SUDEP.pdf (accessed on 20/03/2020)

22. Codes I. 2020 ICD-10-CM Diagnosis Code R56.9: Unspecified convulsions . Icd10data.com. 2019https://www.icd10data.com/ICD10CM/Co des/R00-R99/R50-R69/R56-/R56.9 (accessed 20/03/2020)

23. Common cause in sudden death syndromes. Science Daily. 2019. https://www.sciencedaily.com/releases/2019/03/ 190321142003.htm (accessed on 20/03/2020) 